\title{
ChemComm
}

Check for updates

Cite this: Chem. Commun., 2021, 57,10819

Received 3rd August 2021

Accepted 20th September 2021

DOI: $10.1039 / d 1 c c 04241 f$

rsc.li/chemcomm

\section{Photoswitches for controllable RNA binding: a future approach in the RNA-targeting therapy}

\begin{abstract}
Daria V. Berdnikova (D)
RNA is an emerging drug target that opens new perspectives in the treatment of viral and bacterial infections, cancer and a range of so far incurable genetic diseases. Among the various strategies towards the design and development of selective and efficient ligands for targeting and detection of therapeutically relevant RNA, photoswitchable RNA binders represent a very promising approach due to the possibility to control the ligand-RNA and protein-RNA interactions by light with high spatiotemporal resolution. However, the field of photoswitchable RNA binders still remains underexplored due to challenging design of lead structures that should combine high RNA binding selectivity with efficient photochemical performance. The aim of this highlight article is to describe the development of photoswitchable noncovalent RNA binders and to outline the current situation and perspectives of this emerging interdisciplinary field.
\end{abstract}

\section{Introduction}

The ribonucleic acid (RNA) is the most diverse and multifunctional biomolecule in Nature that not only encodes genetic information, but also catalyses chemical reactions in the cell and provides intracellular recognition and transport. ${ }^{1-3}$ Due to the multiple and important roles of RNA in living processes it

Universität Siegen, Organische Chemie II, Adolf-Reichwein-Str. 2, 57076 Siegen, Germany.E-mail: berdnikova@chemie-bio.uni-siegen.de

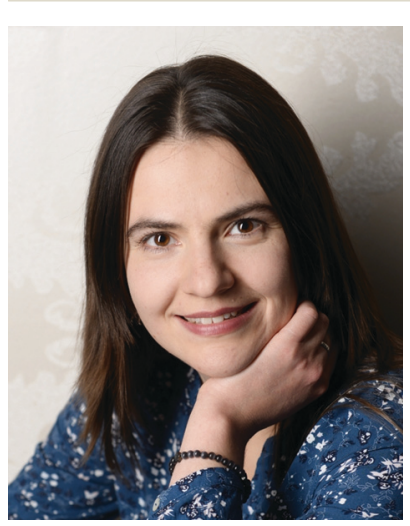

Daria V. Berdnikova

Dr Daria V. Berdnikova obtained her Chemical Engineer degree (2009) with specialization in nanomaterials at D. I. Mendeleev University of Chemical Technology of Russia (Moscow, Russia). In 2012, she received her $P h D$ in organic chemistry and physical chemistry at A. N. Nesmeyanov Institute of Organoelement Compounds of Russian Academy of Sciences (INEOS RAS). In 20132017, she worked as a senior researcher at INEOS RAS. In

2017, she moved to the University of Siegen (Germany), as a Marie Skłodowska Curie fellow. Since 2020, she is a leader of the independent DFG project at the University of Siegen. Her research interests include design and synthesis of DNA- and RNA-targeting ligands, controllable interactions of photoactive molecules with nucleic acids and supramolecular assemblies. has as a huge and essential impact on human health. Structural changes and failure of the function of human RNAs often have significant health-related consequences. For example, more than 40 incurable neurodegenerative diseases, e.g. Huntington disease, are caused by formation of mutant RNAs in humans due to expansions of RNA sequence repeats. ${ }^{4,5}$ Moreover, the involvement of microRNAs (miRNA) in oncogenesis and in the development of metabolic, autoimmune and other disorders was recently discovered. ${ }^{6-8}$ Exogenous RNAs can also pose a serious threat to human health. The majority of human and animal pathogenic viruses are RNA viruses whose genetic information is stored in the form of RNA. These viruses cause a range of diseases such as influenza, measles, polio, rabies, Ebola fever, HIV infection, hepatitis C (HCV), COVID-19 and others. ${ }^{9-11}$ Before this background, RNA obviously represents a promising therapeutical target for treatment of a wide range of diseases, from genetically caused disorders and cancer to lifethreating infections. RNA-targeting therapy could be applied also to bacterial infections because interaction of drugs with ribosomal RNA of bacteria can be used to fight pathogenic microorganisms. ${ }^{12}$ Along these lines, RNA-targeting therapy represents significant expansion, alternative or supplement to the majority of the existing therapeutical approaches that typically target proteins. ${ }^{13}$ The rapidly increasing amount of publications on RNA-targeting molecules points out the emerging importance of this interdisciplinary research area. ${ }^{14-19}$ Similar to DNA and proteins, RNA possesses a range of important features as a drug target. Like DNA, the chemical building units of RNA are fewer and less complex in comparison to those of proteins. RNA can also adopt a regular A-form double-helix, which, however, is often disrupted by regions of unpaired or mismatched bases that allow RNA to fold into more complex 
three-dimensional structures, analogous to those observed in proteins. The plethora of RNA structural elements and folding patterns $^{20}$ that result in the formation of binding sites of unique size, shape, and electrostatic potential distribution is a prerequisite for highly selective interactions of RNA with small molecules. The targetability of RNA arises also from the absence of cellular RNA repair mechanisms and its accessibility in ribonucleoprotein complexes. ${ }^{21}$ Nevertheless, the rational design of selective and efficient RNA binders still represents a challenging task due to the absence of clear and universal design strategies arising from high variability of RNA structures.

One of the major challenges in the development of RNAtargeting drugs is to establish complete spatial and temporal control of the drug activity, specifically the rate of drug delivery, its effective concentration in the proximity of a target, the actual drug-target interaction, as well as the final drug deactivation process. The latter is especially important to suppress potential side effects of the therapy. In this context, the introduction of a switchable functionality into an RNA binder that allows manipulation of its properties by an external stimulus is highly attractive. Among potential external stimuli for drug activation, light is particularly attractive as a noninvasive, bio-orthogonal tool possessing a high spatiotemporal resolution of action. In this context, two main approaches of the photochemical control of the structure and functions of nucleic acids can be applied. ${ }^{22}$ (i) With the first approach, the photoregulation is accomplished by a covalent integration of photoswitches into the nucleic acids. ${ }^{2-25}$ This method is very effective, but suffers from the disturbance of the native structure of nucleic acids, necessity of synthetically challenging modifications of nucleotides and potential difficulties of application in real systems, e.g. due to degradation by nucleases. (ii) The second approach considers the application of photoswitchable ligands as noncovalent nucleic acids binders, whose binding affinity can be regulated by light. Along these lines, a range of noncovalent photocontrollable DNA binders has been described that can be activated for binding interaction with DNA in a reversible ${ }^{26}$ or irreversible ${ }^{27}$ manner. At the same time, the field of photocontrollable noncovalent RNA binders has not been explored to the same extent, most likely, due to the ambiguity of the design strategies. Nevertheless, the accumulated data already allow to perform the first systematization of the noncovalent photoswitchable RNA binders, make some conclusions and suggest new ideas. This highlight outlines the achievements, challenges and future perspectives of the development of noncovalent light-controllable RNA binders with the aim to bring this emerging interdisciplinary area to the attention of chemists and biologists.

\section{Types of the noncovalent photoswitch-RNA interactions}

Generally, there are four types of manipulation of noncovalent ligand-RNA interactions by light (Scheme 1). The first type is a)

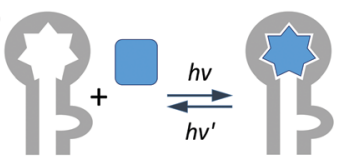

ON/OFF binding

b)

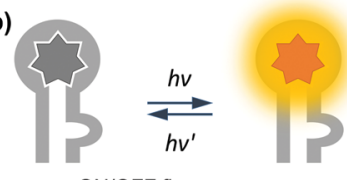

c)

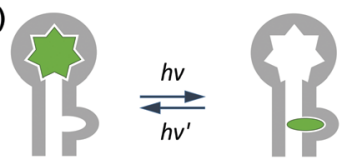

switching of the binding mode

d)

RNA un-/re-folding

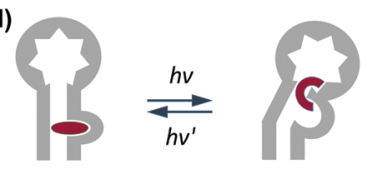

Scheme 1 Existing and prospective types of systems involving noncovalent photoswitch-RNA interactions.

based on the photochemical transformation between two ligand forms that possess significantly different affinity towards RNA. In an ideal scenario, the light-controllable switching between the binding and non-binding isomer yields an "ON/OFF binding" system (Scheme 1a). Hence, the geometrical match of only one of the isomers to the binding pocket of the target RNA is an essential feature of these systems.

In the other three types of the photoswitch-RNA interactions, both the initial and photoinduced forms of the ligand remain bound to the nucleic acid, but the photoswitching allows to manipulate certain binding parameters of the ligand or/and RNA. Thus, the photoswitching can be accompanied by pronounced changes of the ligand fluorescence ("ON/OFF fluorescence") (Scheme 1b), ${ }^{28}$ resulting in a photoswitchable stain, which can be applied in RNA bioimaging. ${ }^{29}$ Other possible effects may be the light-induced change of the ligand binding mode (Scheme 1c) and the light-controllable reversible un- or refolding of RNA (Scheme 1d). The photoinduced switching of the binding mode has been recently described for quadruplex DNA. $^{26 n}$ To the best of my knowledge, however, similar examples for RNA species have not been reported, so far. Nevertheless, such systems can be envisaged for the naturally occurring RNA motifs of sufficient complexity, for example, three-helix junctions, riboswitches and pseudoknots, ${ }^{13}$ as well as RNA G-quadruplexes. ${ }^{30}$

In the next sections, the photoswitchable RNA binders reported up to date are highlighted. The majority of the systems belong to the "ON/OFF binding" type. Two single examples represent the "ON/OFF fluorescence" and "RNA un/re-folding", systems, respectively. The article is divided into sections according to the structure and therapeutical importance of RNA species, namely from synthetic RNAs having low therapeutical relevance to HIV-1 genome RNA.

\section{Photoswitchable RNA binders}

\subsection{Photoswitchable binders for synthetic RNA homoduplexes and cellular double-stranded RNAs}

RNA homoduplexes are RNA double helixes having precisely complementary bases in the two strands. Synthetic RNA homoduplexes are usually composed of two complementary homopolymeric single 
strands, e.g. polyriboadenylic acid (polyA) with polyribouridylic acid (polyU) or polyriboguanilic acid (polyA) with polyribocytidylic acid (polyC). Given to their availability, these RNAs are widely used in fundamental and medicinal studies of nucleic acids. The effect of synthetic RNA homoduplexes to promote antitumoral immune response has been explored in cancer immunotherapy for decades. ${ }^{31}$ However, as a drug target mimetic, such RNAs represent only a very simplifying model, whose structure and properties cannot be directly related to naturally occurring RNAs possessing much more variable composition and folding patterns. Nevertheless, studies with synthetic RNA homoduplexes often become the first step in the evaluation of the RNA-binding properties of ligands. These RNAs possess a highly ordered helical structure with a very shallow minor groove and a very deep and narrow major groove. As a consequence, binding of small ligands to polymeric doublestranded RNA (dsRNA) occurs mainly in two modes, namely intercalation between RNA base pairs and groove binding, like in the case of the double-stranded DNA (dsDNA). For this reason, it is not surprising that most DNA-binding ligands also associate with synthetic RNA homoduplexes.

The first example of the photoactivated noncovalent RNA binding was reported in 2005. ${ }^{27 a}$ Thus, E-methyl-3-(furan-2-yl)2-phenylacrylate $\mathbf{1}$ that has no affinity towards nucleic acids was photochemically transformed into polycyclic compound 2 that intercalates between the dsDNA/RNA base pairs (Scheme 2a). The photoreaction was performed directly in the presence of the double-stranded calf thymus DNA (ct DNA) or synthetic RNA homoduplexes polyA-polyU and polyG-polyC. However, intercalator 2 showed no selectivity towards a certain type of nucleic acids. Additionally, the photoreaction was irreversible, i.e. subsequent photochemical or thermal deactivation of intercalator 2 was not possible.

Very recently, it was reported about the photoswitchable diarylethene-pyrene conjugate $3 \mathbf{o}$ (Scheme $2 \mathrm{~b}$ and Table 1 ) that can be reversibly activated towards binding with polyA-polyU RNA and different types of double-stranded DNA (ct DNA, poly(dA:dT), poly(dG:dC)). ${ }^{32}$ The open form of 30 is accommodated in the dsDNA/RNA groove, whereas intramolecular stacking of two pyrene residues makes the closed form $3 \mathbf{c}$ too bulky for the groove binding resulting in the dissociation of the ligand-RNA complexes.

Another recent example of the photocontrollable interaction with dsRNA is spiropyran derivative 4 (Scheme $2 \mathrm{c}$ and Table 1). ${ }^{33}$ The closed form $4 \mathbf{c}$ did not bind to dsRNA whereas the open form 40 intercalated between the base pairs of the RNA A-helix. Studies with synthetic RNA homoduplexes polyApolyU and polyG-polyC revealed that 40 had a slight preference towards interaction with GC-pairs (Table 1). Moreover, it was shown that the open form $\mathbf{4 0}$ associated with cellular dsRNAs obtained from HeLa cells. The long endogenous dsRNAs were isolated by the treatment of the total HeLa RNA samples with RNase $\mathrm{T} 1$ followed by phenol extraction that allowed to remove single-stranded RNAs and hairpin RNAs. The binding of 40 to the cellular dsRNAs was detected photometrically. However, the identification of the particular structure of the dsRNA species involved in this interaction has not been performed. a) (c)

1

no DNARNA binding
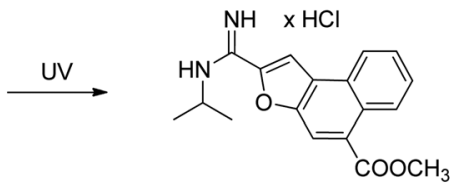

2

dsDNA/dsRNA intercalator

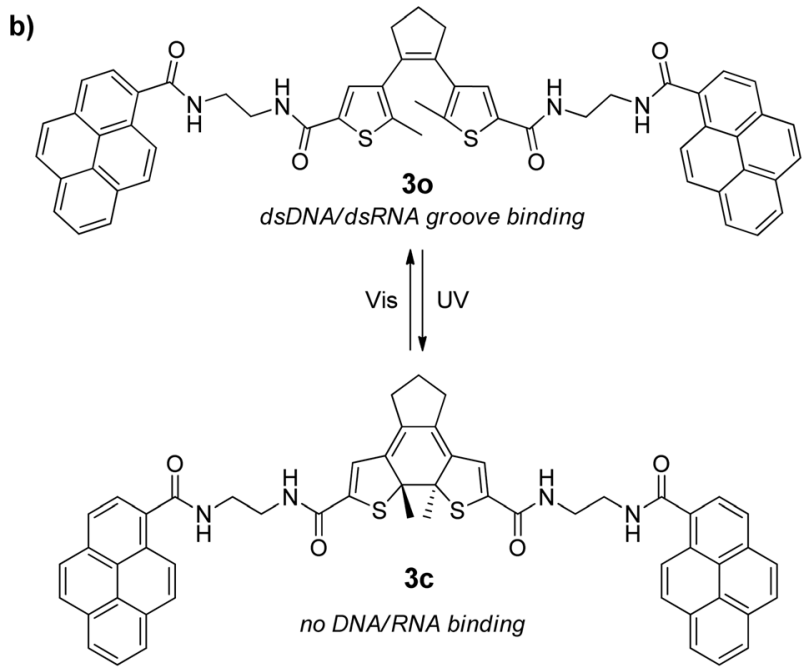

c)
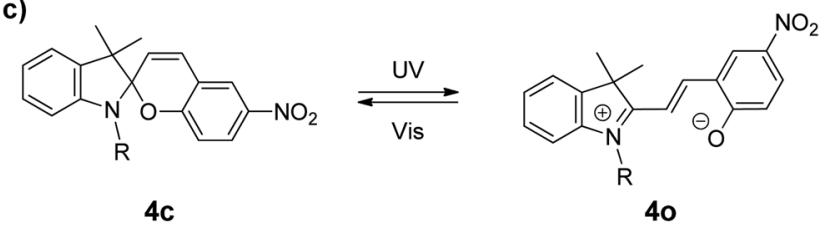

no dsRNA binding

RNA aptamer binding

dsRNA intercalator no RNA aptamer binding

Scheme 2 Photoswitchable binders for double-stranded RNAs (compound $\mathbf{4}$ also interacts with an RNA aptamer, see Section 3.2).

Additionally, the open form of spiropyran 40 was applied for the optical detection of the increased dsRNA expression in the HCT116 colorectal cancer cells after treatment with anticancer drug decitabine. ${ }^{33}$ The analysis was performed on the isolated dsRNAs samples.

Overall, the association of small ligands with dsRNA homopolymers and long cellular dsRNAs does not significantly differ from the interaction with polymeric dsDNA. For this reason, photoswitchable ligands 1, 3 and 4 lack selectivity in discrimination of dsRNA against dsDNA.

\subsection{Photoswitchable binders for RNA aptamers}

The next approximation towards native RNA structures was realized by photocontrollable interactions with RNA aptamers, i.e. short single-stranded RNAs that can selectively associate with a specific target. ${ }^{34-36}$ The high affinity and selectivity of 


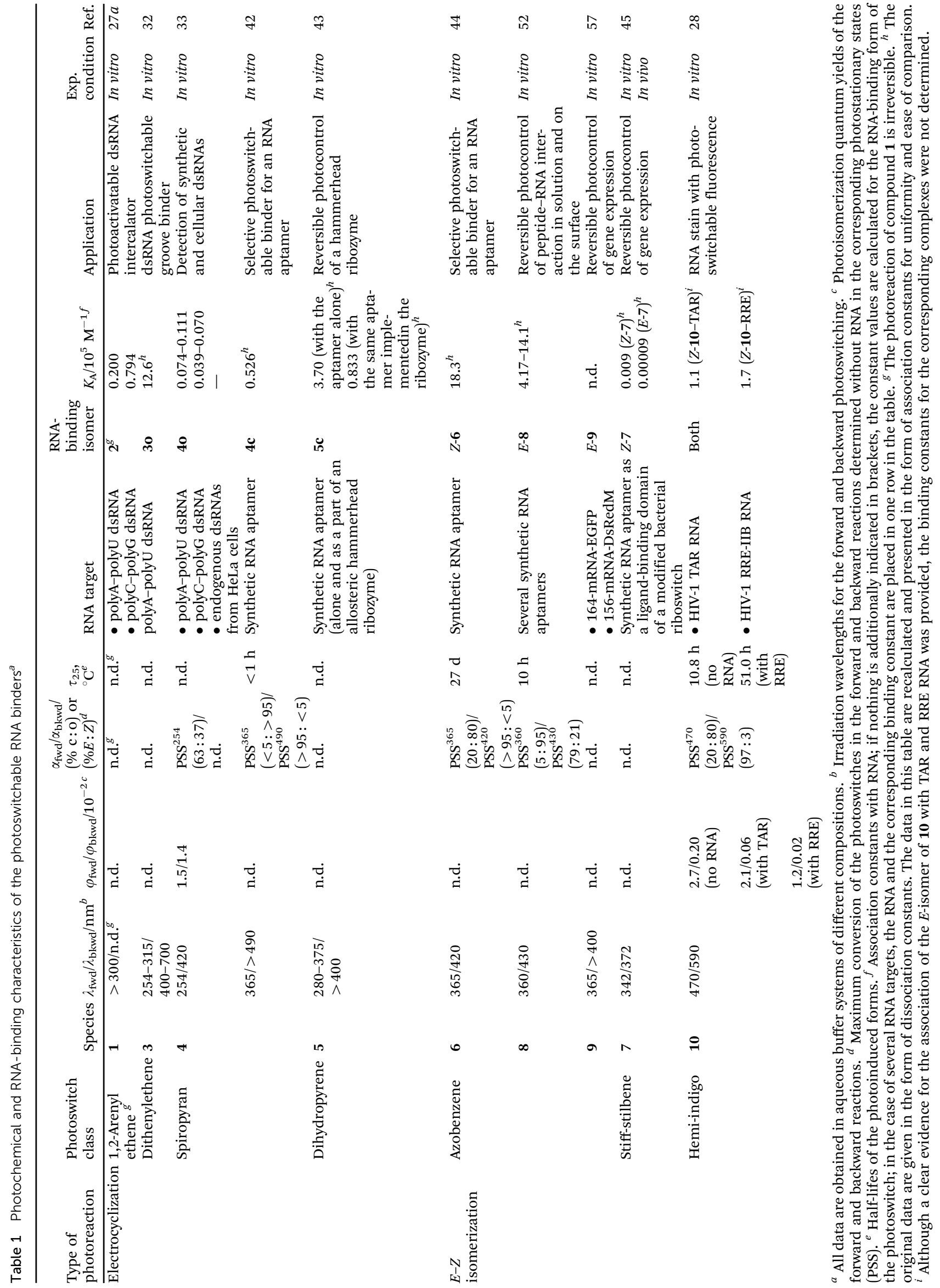


the aptamer-target binding is provided by the unique tertiary structure of aptamers arising from their flexibility and shapeforming properties. ${ }^{37}$ To date, a wide range of aptamers has been obtained for various targets, from small organic and inorganic molecules to protein complexes and even cells. ${ }^{38}$ In medicine, aptamers designed for certain therapeutic targets are used as analogues of antibodies for diagnostic and treatment purposes. ${ }^{39-41}$ RNA aptamers represent a more realistic model of native RNAs in comparison to the synthetic RNA homoduplexes. However, the majority of RNA aptamers obtained up to now does not correspond to the native RNA sequences, although they can be artificially implemented, for example, into bacterial plasmids.

The peculiarities of the aptamer-ligand interactions allowed to obtain systems with highly efficient, light-controllable switching of the ligand binding state ("ON/OFF binding"). Thus, four examples of photoswitchable binders for RNA aptamers were reported that are based on the reversible photoinduced transformations of spiropyran $4,{ }^{42}$ benzodihydropyrene $5,{ }^{43}$ azobenzene $6,{ }^{44}$ and stiff-stilbene $7^{45}$ (Schemes 2c, 3 and Table 1). The RNA aptamers that bind specifically only to one of the two forms of the ligands 4-6 were selected in vitro from RNA aptamer libraries by the Systematic Evolution of Ligands by EXperimental enrichment (SELEX) procedure ${ }^{46}$ i.e. by screening the geometrical fit of the ligands to the aptamer binding pockets. In the case of stiffstilbene 7, the selection was performed using an RNA pool derived from a bacterial SAM-1 riboswitch by replacing its ligand-binding domain with a random region of 45 nucleotides and partially randomizing the terminator and anti-terminator hairpins. ${ }^{45}$ As a result, the selected RNA aptamer was isolated as a fused construct together with a riboswitch expression platform. The reversible photoswitching between the isomers of ligands 4-7 gave the systems an advantage of accomplishing repeated binding/dissociation cycles with RNA. Whereas the studies on spiropyran 4 and azobenzene 6 derivatives provided a proof-of-principle for the selective light-controllable RNA binding, compounds 5 and 7 were used in more complex systems for the manipulation of the biological functions. Thus, introduction of the corresponding RNA aptamer into a hammerhead ribozyme yielded a two-state system with a >900-fold difference in catalytic activity that was governed by the photoswitchable interaction with dihydropyrene derivative $5 .{ }^{43}$ In the second example, a synthetic riboswitch comprising the stiff-stilbene 7-binding aptamer has been successfully implemented into a bacterial plasmid that allowed to perform reversible photocontrol of the Fluc gene expression in the modified $E$. coli in vivo. ${ }^{45}$

Nevertheless, all the studied aptamer models were selected from the randomized RNA libraries and had, therefore, no therapeutic relevance, thus reducing the applicability of these systems for real human disease models.

\subsection{Special case: photoswitchable peptide-RNA interactions}

The investigation of photocontrollable protein-RNA interactions is especially important for a therapeutic perspective because aberrant protein-RNA interactions are involved in pathogenic mechanisms of various diseases. These interactions

a)

a) $\mathrm{HOOC}$
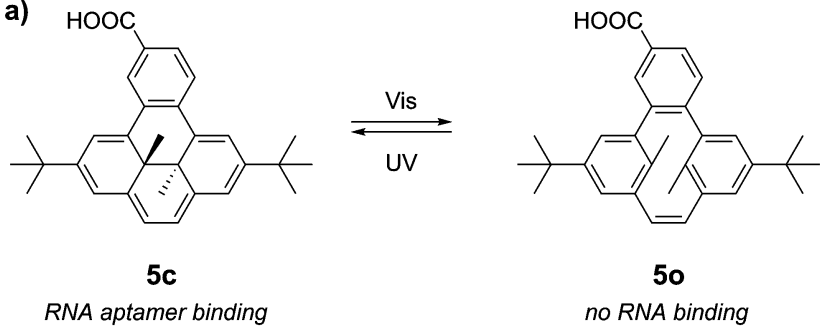

50

no RNA binding

b)

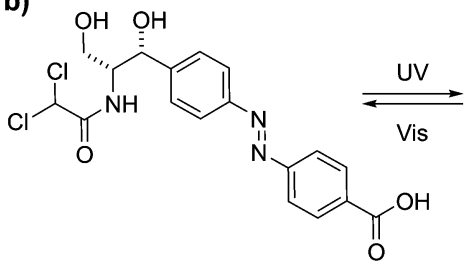

Z-6

RNA aptamer binding

c)

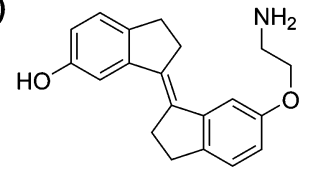

Z-7

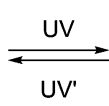

RNA aptamer binding

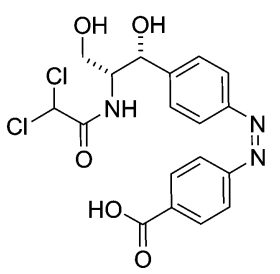

E-6

no RNA binding
Scheme 3 Photoswitchable binders for RNA aptamers.

involve abnormal protein binding to endogenous mutant RNAs, which happens, for example, in RNA repeat expansion diseases. ${ }^{16}$ In the case of viral infections, association of certain proteins to viral genome RNA plays an important role in the living cycle of viruses. For example, binding of the Tat protein to the transactivation response element (TAR) of the human immunodeficiency virus (HIV) RNA triggers the transcription of HIV in infected cells. ${ }^{47}$ In this context, targeted disruption of disease-related protein-RNA complexes is critical for achieving a therapeutic effect in the treatment of RNA-associated diseases. Along these lines, reversible regulation of protein-RNA interactions by light may act not only as a therapeutical approach but also as a powerful model for the understanding of molecular mechanisms that underlie corresponding pathogenic processes.

Although the range of light-responsive peptides has been developed for peptide-DNA-interactions, ${ }^{48-51}$ the photoswitchable RNA-targeting peptides have been, again, surprisingly underexplored. The first and, so far, the only fully lightcontrollable peptide-RNA interaction was accomplished by the introduction of the photoswitchable azobenzene moiety into a short peptide. ${ }^{52}$ Several RNA aptamers that selectively bind to the photoresponsive peptide 8 in its $E$-form (Scheme 4 and Table 1) were obtained by in vitro selection from 70 random nucleotides sequence pool of RNA. The $E-Z$ photoisomerization 

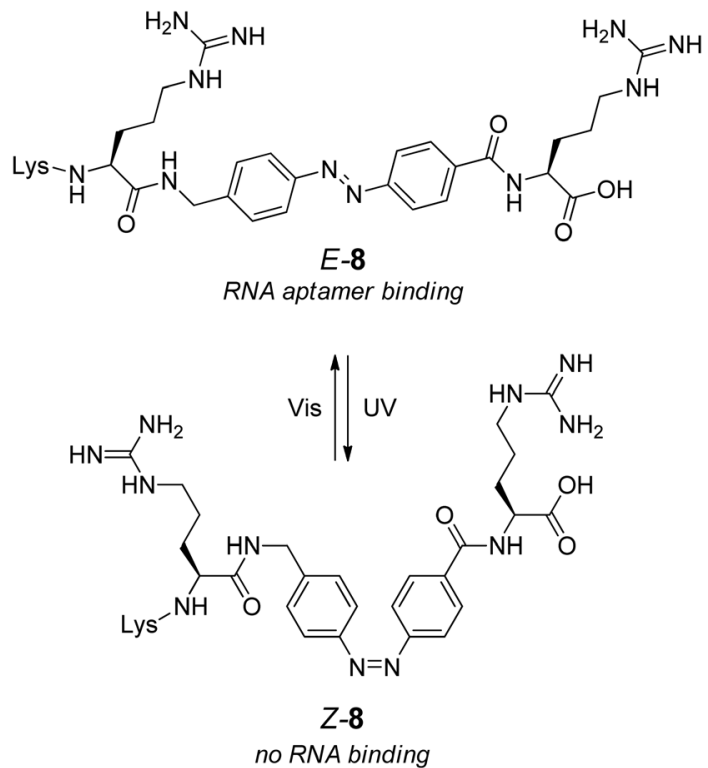

Scheme 4 Photoswitchable azobenzene-derivatized peptide for the RNA aptamer binding.

of the azobenzene chromophore upon UV irradiation resulted in significant changes in the structure of peptide $Z-8$ leading to the dissociation of the peptide-RNA complex. The backwards $Z-E$ isomerization and re-activation of the peptide $E-\mathbf{8}$ towards RNA binding was performed upon irradiation with visible light.

Overall, the studies with synthetic RNA homoduplexes and aptamers provided a clear proof-of-principle for the "ON/OFF binding" concept to control ligand-RNA interactions by light. Nevertheless, these studies were mainly performed on nonnative RNA sequences or undefined natural RNAs that may not be appropriate models for the therapeutically relevant human or viral RNAs. Application of the UV light for the photoswitching in model systems is, in principle, justified because RNA is more UV-resistant in comparison with DNA. ${ }^{53}$ However, in real systems the necessity to use UV light for the activation of the binders may significantly hamper their potential application due to the damage of biomolecules and low UV penetration depth of the tissues. ${ }^{54}$

\subsection{Photoswitchable binders for messenger RNAs (mRNAs)}

A messenger RNA (mRNA) is a single-stranded ribonucleic acid that is complementary to one of the DNA strands of a gene. ${ }^{2}$ The genetic information on the protein structure is copied from DNA into an mRNA molecule during the transcription process. Once it is complete, the mRNA moves to the cytoplasm where ribosomes decode it and synthesize the corresponding protein during the translation process. The importance of mRNA studies has recently drawn public attention in connection with the application of mRNA-based vaccines for the immunization against COVID-19 that started in December 2020. ${ }^{55,56}$

Despite the large amount of publications on mRNA, there is only one example of the interaction of an organic photoswitch with mRNA, so far. ${ }^{57}$ Thus, a positively charged azobenzene

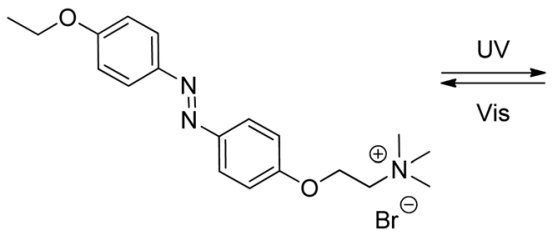

E-9

mRNA condensation

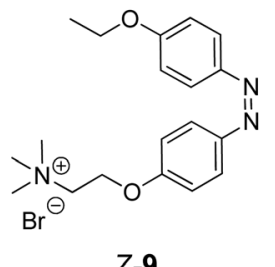

mRNA de-condensation

Scheme 5 Photoswitchable binder for mRNAs.

derivative $E$-9 (Scheme 5 and Table 1) that acts as anspecific electrostatic binder induced aggregation of 759-base mRNA coding the Enhanced Green Fluorescent Protein (EGFP) ${ }^{58}$ in the in vitro experiments. ${ }^{57 a}$ The system represents the "RNA un/re-folding" principle (Scheme 1d). Since the protein translation directly depends on the condensation state of the nucleic acid, mRNA aggregation yielded the inhibition of the EGFP synthesis. The $E-Z$ photoisomerization of $E-\mathbf{9}$ upon irradiation with $365 \mathrm{~nm}$ light triggered the dissociation of the mRNA aggregates and re-activated the translation process. The inhibition and re-activation of the translation was followed by the detection of the fluorescence intensity of EGPF. In a follow-up study, ${ }^{57 b}$ the $E$-9-induced condensation of mRNAs was accomplished in the presence of gene-silencing small RNAs. Two mRNAs called 164-mRNA-EGFP and 156-mRNA-DsRedM, which code EGFP and DsRedM ${ }^{59}$ fluorescent proteins and additionally contain antisense sequences of two small RNAs (miR164 and miR156), respectively, were used in this experiment. In this case, the $E-Z$ isomerization of $E-9$ led to the selective photoactivation of genes that were not silenced by small RNAs.

\subsection{Photoswitchable binders for natural therapeutically relevant RNAs}

The first example of a noncovalent photoactive ligand for natural therapeutically relevant RNA was reported in $2019 .^{28}$ In this study, a photoswitchable hemi-indigo binder Z-10 for human immunodeficiency virus RNA was developed. It was shown that compound Z-10 (Scheme 6 and Table 1) associated with the transactivation response element (TAR) and the stem IIB region of the Rev response element (RRE-IIB) of HIV-1 RNA. In contrast to the previously reported systems, both forward and backward photoisomerization of $\mathbf{1 0}$ could be activated by visible light and did not require the use of UV. However, both the initial Z-10 and photoinduced $E-\mathbf{1 0}$ forms showed

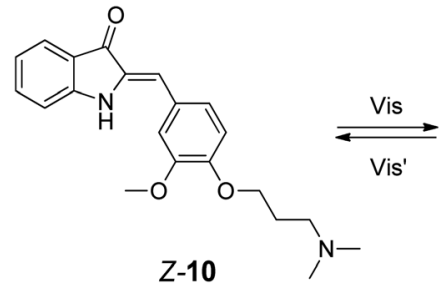

HIV RNA binding fluorescence ON

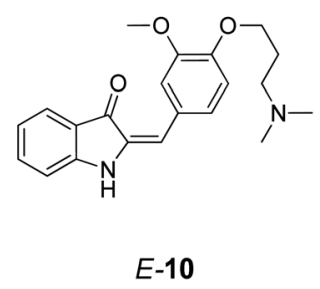

HIV RNA binding fluorescence OFF
Scheme 6 Photoswitchable binder for HIV-1 TAR and RRE-IIB RNA. 
significant affinity towards RNA, thus hampering the photoregulation of binding. Nevertheless, photoswitching of the RNA-bound Z-10 resulted in significant changes in the fluorescent properties of the ligand which allowed to turn the fluorescent staining of RNA on (Z-10-RNA) and off ( $E$-10-RNA) by repeated irradiation with blue and amber light ("ON/OFF fluorescence"). On/off switching of the fluorescence could be performed only upon binding of $\mathbf{1 0}$ to RNA because the ligand showed no emission in bulk solution. In this context, binding of both isomers of $\mathbf{1 0}$ to RNA is advantageous because the ligand can be used as a tunable fluorescent stain to study dynamic processes of the RNA.

\section{Summary and outlook}

To sum up, the approaches towards development of photocontrollable interactions with RNA are far from being fully explored and realized. This is not surprising because the design of photoswitchable RNA binders is particularly challenging due to the limited possibility of the ligand modification. Thus, structural modifications for adjustment of the RNA binding properties can be mainly performed on the binder periphery keeping the photoswitchable core intact such that the photochemical properties are not disrupted. Additionally, for the realization of the "ON/OFF binding", only one of the two isomers of the photoswitch should possess a strong affinity towards RNA to ensure the photocontrol of the binding. For this reason, in the majority of cases, it was the RNA structure that was selected from the pool to bind selectively to a photoswitch with known structure. The only attempt to target predefined therapeutically relevant sequences (TAR and RRE-IIB HIV RNA) with a photoswitchable ligand yielded the RNA binder $\mathbf{1 0}$ of "ON/OFF fluorescence" type. However, the selectivity towards a certain RNA structure as well as the photocontol of binding to naturally occurring RNAs still was not achieved.

A significant potential of photoswitchable ligands for controllable binding and manipulation of the spatial structure of therapeutically relevant RNAs, including RNA repeat expansions is foreseen. Light-controllable manipulation of the RNA folding will, in turn, open the opportunity of reversible regulation of the RNA-protein interactions for investigation and treatment purposes. Additionally, selective RNA markers with photoswitchable fluorescence are perspective for the realtime monitoring of RNA dynamics by novel bioimaging approaches. ${ }^{29}$ Interestingly, all the examples highlighted in this article were developed experimentally without the use of any computational methods and modelling. Along these lines, future application of computational approaches that facilitate the design of photoswitchable RNA binders of different types (Scheme 1) is envisaged. ${ }^{60}$ As have been shown by collected examples, different classes of photoswitches are compatible with RNA binding (Table 1). However, for real applications, special attention should be paid to the shifting of the excitation energy to longer wavelength that, in an ideal case, should fit to the biooptical transparency window $(650-900 \mathrm{~nm}) .{ }^{54}$ In this context, all-visible indigoid photoswitches, such as hemiindigo, ${ }^{61}$ hemithioindigo $^{61}$ and $N, N^{\prime}$-substituted indigo, ${ }^{62}$ represent promising candidates for the further development of the photoswitchable binders for disease-related RNA species. Overall, I hope that this highlight will provide a basis for the development of various photoswitchable RNA binders for future therapeutical applications.

\section{Conflicts of interest}

There are no conflicts to declare.

\section{Acknowledgements}

Generous financial support from Deutsche Forschungsgemeinschaft (project BE 7202/1-1) is gratefully acknowledged. I thank Prof. Dr Heiko Ihmels (Universität Siegen, Germany), Dr Anna Berdnikova (Moscow State University of Medicine and Dentistry, Russia) and Dr Ekaterina Chernikova (INEOS RAS, Russia) for fruitful discussions and valuable advices.

\section{Notes and references}

1 RNA Worlds: From Life's Origins to Diversity in Gene Regulation, ed. P. V. Peplow, B. Martinez, G. A. Calin and A. Esquela-Kerscher, Cold Spring Harbor Laboratory Press, New York, 5th edn, 2019.

2 Nucleic Acids in Chemistry and Biology, ed. G. M. Blackburn, RSC Publishing, Cambridge, 3rd edn, 2006.

3 P. A. Sharp, Cell, 2009, 136, 577.

4 H. Paulson, Handb. Clin. Neurol., 2018, 147, 105.

5 V. Bernat and M. D. Disney, Neuron, 2015, 87, 28.

6 MicroRNAs in Diseases and Disorders: Emerging Therapeutic Targets, ed. J. F. Atkins, R. F. Gesteland and T. R. Cech, RSC Publishing, Cambridge, 2019.

7 S. M. Hammond, Curr. Opin. Genet. Dev., 2006, 16, 4.

8 T. A. Farazi, J. I. Spitzer, P. Morozov and T. Tuschl, J. Pathol., 2011, 223, 102.

9 E. J. Lefkowitz, D. M. Dempsey, R. C. Hendrickson, R. J. Orton, S. G. Siddell and D. B. Smith, Nucleic Acids Res., 2018, 46, D708.

10 E. D. Holmes, The Evolution and Emergence of RNA Viruses, Oxford University Press, New York, 2009.

11 S. J. Russell, Cancer Gene Ther., 2002, 9, 961.

12 D. N. Wilson, Nat. Rev. Microbiol., 2014, 12, 35.

13 K. D. Warner, C. E. Hajdin and K. M. Weeks, Nat. Rev. Drug Discovery, 2018, 17, 547.

14 J. P. Falese, A. Donlic and A. E. Hargrove, Chem. Soc. Rev., 2021, 50, 2224.

15 S. M. Meyer, C. C. Williams, Y. Akahori, T. Tanaka, H. Aikawa, Y. Tong, J. L. Childs-Disney and M. D. Disney, Chem. Soc. Rev., 2020, 49, 7167.

16 A. Ursu, J. L. Childs-Disney, R. J. Andrews, C. A. O'Leary, S. M. Meyer, A. J. Angelbello, W. N. Moss and M. D. Disney, Chem. Soc. Rev., 2020, 49, 7252.

17 M. D. Disney, J. Am. Chem. Soc., 2019, 141, 6776.

18 RNA Therapeutics, ed. A. L. Garner, Springer, Cham, 2018.

19 J. R. Thomas and P. J. Hergenrother, Chem. Rev., 2008, 108, 1171.

20 J. Nowakowski and I. Tinoco Jr, Semin. Virol., 1997, 8, 153.

21 A. R. Ferré-D'Amaré and J. A. Doudna, Annu. Rev. Biophys. Biomol. Struct., 1999, 28, 57.

22 (a) A. S. Lubbe, W. Szymanski and B. L. Feringa, Chem. Soc. Rev., 2017, 46, 1052; (b) W. Szymanski, J. M. Beierle, H. A. V. Kistemaker, W. A. Velema and B. L. Feringa, Chem. Rev., 2013, 113, 6114.

23 Z. L. Pianowski, Chem. - Eur. J., 2019, 25, 5128.

24 M. You and S. R. Jaffrey, Ann. N. Y. Acad. Sci., 2015, 1352, 13.

25 (a) K. Murayama, Y. Yamano and H. Asanuma, J. Am. Chem. Soc., 2019, 141, 9485; (b) Y. Kamiya, Y. Arimura, H. Ooi, K. Kato, 
X.-G. Liang and H. Asanuma, ChemBioChem, 2018, 19, 1305; (c) T. Doi, H. Kashida and H. Asanuma, Org. Biomol. Chem., 2015, 13, 4430; (d) Y. Kamiya and H. Asanuma, Acc. Chem. Res., 2014, $47,1663$.

26 (a) C. Dohno, S. Uno and K. Nakatani, J. Am. Chem. Soc., 2007, 129, 11898; (b) J. Andersson, S. Li, P. Lincoln and J. Andréasson, J. Am. Chem. Soc., 2008, 130, 11836; (c) C. Dohno, T. Yamamoto and K. Nakatani, Eur. J. Org. Chem., 2009, 4051; (d) X. Wang, J. Huang, Y. Zhou, S. Yan, X. Weng, X. Wu, M. Deng and X. Zhou, Angew. Chem., Int. Ed., 2010, 49, 5305; (e) X. Xing, X. Wang, L. Xu, Y. Tai, L. Dai, X. Zheng, W. Mao, X. Xu and X. Zhou, Org. Biomol. Chem., 2011, 9, 6639; $(f)$ A. Mammana, G. T. Carroll, J. Areephong and B. L. Feringa, J. Phys. Chem. B, 2011, 115, 11581; $(g)$ S. V. Paramonov, V. Lokshin, H. Ihmels and O. A. Fedorova, Photochem. Photobiol. Sci., 2011, 10, 1279; (h) H. Ihmels, J. Mattay, F. May and L. Thomas, Org. Biomol. Chem., 2013, 11, 5184; (i) M. Hammarson, J. R. Nilsson, S. Li, P. Lincoln and J. Andréasson, Chem. - Eur. J., 2014, 20, 15855; $(j)$ M. P. O'Hagan, S. Haldar, M. Duchi, T. A. A. Oliver, A. J. Mulholland, J. C. Morales and M. C. Galan, Angew. Chem., Int. Ed., 2019, 58, 4334; (k) B. Heinrich, K. Bouazoune, M. Wojcik, U. Bakowsky and O. Vázquez, Org. Biomol. Chem., 2019, 17, 1827; (l) S. Müller, J. Paulus, J. Mattay, H. Ihmels, V. I. Dodero and N. Sewald, Beilstein J. Org. Chem., 2020, 16, 60; $(m)$ S. Kölsch, H. Ihmels, J. Mattay, N. Sewald and B. O. Patrick, Beilstein J. Org. Chem., 2020, 16, 111; (n) M. P. O'Hagan, J. Ramos-Soriano, S. Haldar, S. Sheikh, J. C. Morales, A. J. Mulholland and M. C. Galan, Chem. Commun., 2020, 56, 5186.

27 (a) K. Starčević, G. Karminski-Zamola, I. Piantanida, M. Žinić, L. Šuman and M. Kralj, J. Am. Chem. Soc., 2005, 127, 1074; (b) M. L. Di Pietro, F. Puntoriero, F. Tuyéras, P. Ochsenbein, P. P. Lainé and S. Campagna, Chem. Commun., 2010, 46, 5169; (c) M. I. Sánchez, O. Vázquez, M. E. Vázquez and J. L. Mascareñas, Chem. Commun., 2011, 47, 11107; (d) D. Berdnikova, O. Fedorova, E. Gulakova and H. Ihmels, Chem. Commun., 2012, 48, 4603; (e) D. V. Berdnikova, T. M. Aliyeu, T. Paululat, Y. V. Fedorov, O. A. Fedorova and H. Ihmels, Chem. Commun., 2015, 51, 4906; $(f)$ D. V. Berdnikova, J. Heider, H. Ihmels, N. Sewald and P. M. Pithan, ChemPhotoChem, 2020, 4, 520.

28 (a) D. V. Berdnikova, Chem. Commun., 2019, 55, 8402; (b) D. V. Berdnikova, Beilstein J. Org. Chem., 2019, 15, 2822.

29 (a) Y. Zhang, S. Tang, E. R. Thapaliya, L. Sansalone and F. M. Raymo, Chem. Commun., 2018, 54, 8799; (b) Y. Zhang, S. Tang, L. Sansalone, J. D. Baker and F. M. Raymo, Chem. - Eur. J., 2016, 22, 15027; (c) Y. Xiong, P. Rivera-Fuentes, E. Sezgin, A. V. Jentzsch, C. Eggeling and H. L. Anderson, Org. Lett., 2016, 18, 3666; (d) M. N. Tran and D. M. Chenoweth, Angew. Chem., Int. Ed., 2015, 54, 6442.

30 M. M. Fay, S. M. Lyons and P. Ivanov, J. Mol. Biol., 2017, 429, 2127.

31 For example, see: (a) J. Lacour, A. Spira, J.-Y. Petit, D. Sarrazin, F. Lacour, M. Michelson, G. Delage, G. Contesso and J. Viguier, Lancet, 1980, 316, 161; (b) G. Gatti, N. G. Nuñez, D. A. Nocera, L. Dejager, C. Libert, C. Giraudo and M. Maccioni, Eur. J. Immunol., 2013, 43, 1849.

32 I. Orehovec, M. Matković, I. Pehar, D. Majhen and I. Piantanida, Int. J. Mol. Sci., 2021, 22, 4916.

33 A. A. Ali, M. Kang, R. Kharbash and Y. Kim, BMC Biomed. Eng., 2019, $1,6$.

34 M. R. Dunn, R. M. Jimenez and J. C. Chaput, Nat. Rev. Chem., 2017, 1, 0076.
35 K. N. Kang and Y. S. Lee, RNA Aptamers: A Review of Recent Trends and Applications, in Future Trends in Biotechnology. Advances in Biochemical Engineering/Biotechnology, ed. J. J. Zhong, Springer, Berlin, Heidelberg, 2012, vol. 131.

36 P. Röthlisberger and M. Hollenstein, Adv. Drug Delivery Rev., 2018, $134,3$.

37 T. Adachi and Y. Nakamura, Molecules, 2019, 24, 4229.

38 D. Bunka and P. Stockley, Nat. Rev. Microbiol., 2006, 4, 588.

39 Y. S. Kim, N. H. A. Raston and M. B. Gu, Biosens. Bioelectron., 2016, 76, 2.

40 J. Zhou, M. L. Bobbin, J. C. Burnett and J. J. Rossi, Front. Genet., 2012, 3, 234.

41 A. B. Iliuk, L. Hu and W. A. Tao, Anal. Chem., 2011, 83, 4440.

42 (a) D. D. Young and A. Deiters, ChemBioChem, 2008, 9, 1225; (b) X. Zhang, J. Zhang, Y.-L. Ying, H. Tian and Y.-T. Long, Chem. Sci., 2014, 5, 2642.

43 H. W. Lee, S. G. Robinson, S. Bandyopadhyay, R. H. Mitchel and D. Sen, J. Mol. Biol., 2007, 371, 1163.

44 T. S. Lotz, T. Halbritter, C. Kaiser, M. M. Rudolph, L. Kraus, F. Groher, S. Steinwand, J. Wachtveitl, A. Heckel and B. Suess, Nucleic Acids Res., 2019, 47, 2029.

45 K. A. Rotstan, M. M. Abdelsayed, L. F. M. Passalacqua, F. Chizzolini, K. Sudarshan, A. R. Chamberlin, J. Míšek and A. Luptak, eLife, 2020, 9, e51737.

46 R. Stoltenburg, C. Reinemann and B. Strehlitz, Biomol. Eng., 2007, 24, 381.

47 J. Karn, J. Mol. Biol., 1999, 293, 235.

48 L. Albert and O. Vázquez, Chem. Commun., 2019, 55, 10192.

49 J. Rodriguez, J. Mosquera, S. Learte-Aymamí, M. E. Vázquez and J. L. Mascareñas, Acc. Chem. Res., 2020, 53, 2286.

50 A. A. Beharry and G. A. Woolley, Chem. Soc. Rev., 2011, 40, 4422.

51 V. Peddie and A. D. Abell, J. Photochem. Photobiol., C, 2019, 40, 1.

52 (a) G. Hayashi, M. Hagihara, C. Dohno and K. Nakatani, J. Am. Chem. Soc., 2007, 129, 8678; (b) G. Hayashi, M. Hagihara and K. Nakatani, Chem. - Eur. J., 2009, 15, 424; (c) G. Hayashi and K. Nakatani. Development of Photoswitchable RNA Aptamer-Ligand Complexes, in Artificial Riboswitches. Methods in Molecular Biology (Methods and Protocols), ed. A. Ogawa, Humana Press, Totowa, NJ, 2014, vol. 1111.

53 L. M. Kundu, U. Linne, M. Marahiel and T. Carell, Chem. - Eur. J., 2004, 10, 5697.

54 R. Weissleder, Nat. Biotechnol., 2001, 19, 316.

55 K. S. Park, X. Sun, M. E. Aikins and J. J. Moon, Adv. Drug Delivery Rev., 2021, 169, 137.

56 D. Pushparajah, S. Jimenez, S. Wong, H. Alattas, N. Nafissi and R. A. Slavcev, Adv. Drug Delivery Rev., 2021, 170, 113.

57 (a) A. Estévez-Torres, C. Crozatier, A. Diguet, T. Hara, H. Saito, K. Yoshikawa and D. Baigl, Proc. Natl. Acad. Sci. U. S. A., 2009, 106, 12219; (b) S. Rudiuk, H. Saito, T. Hara, T. Inoue, K. Yoshikawa and D. Baigl, Biomacromolecules, 2011, 12, 3945.

58 B. P. Cormack, R. H. Valdivia and S. Falkow, Gene, 1996, 173, 33.

59 D. E. Strongin, B. Bevis, N. Khuong, M. E. Downing, R. L. Strack, K. Sundaram, B. S. Glick and R. J. Keenan, Protein Eng., Des. Sel., 2007, 20, 525.

60 D. V. Berdnikova, P. Carloni, S. Krauß and G. Rossetti, Molecules, 2021, 26, 3384.

61 C. Petermayer and H. Dube, Acc. Chem. Res., 2018, 51, 1153.

62 C.-Y. Huang, A. Bonasera, L. Hristov, Y. Garmshausen, B. M. Schmidt, D. Jacquemin and S. Hecht, J. Am. Chem. Soc., 2017, 139, 15205. 